were removed to analyse fibrotic changes. As shown in figure $1 \mathrm{a}$, the severity of fibrotic changes as indicated by the Ashcroft score was similar in wildtype and PAR-4 deficient animals (score of mean \pm SEM $3.46 \pm 0.25$ and $2.48 \pm 0.50$, respectively, $p=0.13$ ). In line, collagen deposition as assessed using Masson's trichrome stainings was also similar in wildtype and PAR-4 deficient mice (mean \pm SEM arbitrary units of $2.2 \pm 0.9$ and $2.0 \pm 0.8$, respectively, $\mathrm{p}=0.88$; fig. $1 \mathrm{~b}$ ). Finally, we determined pro-fibrotic gene expression in the lungs of bleomycin treated mice and, as shown in figure 1c, no differences in fibronectin, collagen 1 and collagen 3 levels between wildtype and PAR-4 deficient mice were observed. Overall these data thus indicate that PAR-4 does not play a significant role in the regulation of pulmonary fibrosis. Although extrapolation of our data to other fibrotic disorders should be performed with great care, our data argue against an important role of PAR-4 in fibrotic disease and once more stress that in vitro data should not be over interpreted by claiming potential roles in vivo.

\footnotetext{
Keren Borensztajn ${ }^{*, \#, \text { J JanWillem Duitman }}{ }^{*}$,

Lois W. Brüggemann* and C. Arnold Spek*

*Center for Experimental and Molecular Medicine, Academic Medical Center, Amsterdam, the Netherlands, and "INSERM, U700, University Paris Diderot, Paris, France. ${ }^{\circledR}$ These authors contributed equally.
}

Correspondence: C.A. Spek, Center for Experimental and Molecular Medicine, Academic Medical Center, Room H2-257, Meibergdreef 9, 1105 AZ Amsterdam, The Netherlands E-mail: c.a.spek@amc.uva.nl

Statement of Interest: None declared.

\section{REFERENCES}

1 Macfarlane SR, Seatter MJ, Kanke T, et al. Protease-activated receptors. Phamacol Rev 2001; 53: 245-282.

2 Ando S, Otani H, Yagi Y, et al. Proteinase-activated receptor 4 stimulation-induced epithelial-mesenchymal transition in alveolar epithelial cells. Respir Res 2007; 8: 31.

3 Peters T, Henry PJ. Protease-activated receptors and prostaglandins in inflammatory lung disease. Br J Pharmacol 2009; 158: 1017-1033.

4 Ramachandran R, Noorbakhsh F, Defea K, et al. Targeting proteinase-activated receptors: therapeutic potential and challenges. Nat Rev Drug Discov 2012; 11: 69-86.

5 Borensztajn K, Bresser P, van der Loos C, et al. Protease-activated receptor-2 induces myofibroblast differentiation and tissue factor up-regulation during bleomycin-induced lung injury: potential role in pulmonary fibrosis. Am J Pathol 2010; 177: 2753-2764.

DOI: $10.1183 / 09031936.00045812$

\title{
Severe pulmonary hypertension leading to heart-lung transplantation and revealing breast cancer
}

\section{To the Editors:}

Pulmonary tumour embolism is considered to be a rare cause of pulmonary hypertension. The incidence, studied in autopsy series, varies from 3 to $26 \%$ of patients with solid tumour [1]. It occurs most frequently in breast, stomach, lung, liver, prostate and pancreas adenocarcinoma [1]. The literature shows a male predominance and an average age of $52.4 \mathrm{yrs}$ [2]. The signs are those of any pulmonary hypertension, occurring either during the history of a known neoplasia or as the first manifestation of malignancy. Pre-mortem diagnosis is difficult to confirm and definitive diagnosis is usually made on an autopsy study. Several studies have reported pulmonary wedge aspiration cytology performed during a right heart catheterisation as a useful premortem diagnostic tool [3]. Treatment consists of treating the neoplasia. Specific treatment for pulmonary hypertension has not been evaluated. Most frequently, evolution leads to refractory right cardiac failure and death [4]. In rare cases improvement has been reported after chemotherapy [5] or endarterectomy [6].

Two mechanisms can be involved in the development of pulmonary hypertension in tumour pulmonary embolism: the first one is by mechanical occlusion of the small pulmonary arteries by multiple neoplastic microemboli, the second one is through development of pulmonary tumour thrombotic microangiopathy (PTTM) as a result of endothelial interaction with tumoural cells. In PTTM, inflammatory and coagulation pathways are activated leading to vascular remodelling phenomena and diffuse narrowing of the pulmonary arteriolar system [7]. The role of tissue factor and vascular endothelial growth factor [8] as well as platelet-derived growth factor in the pathogenesis of PTTM has been highlighted by previous studies.

We report the case of a 49 -yr-old female patient who was admitted on suspicion of pulmonary hypertension. This episode began 4 months ago following complaints of exercise-induced dyspnoea. A pulmonary angiographic computed tomography (CT) found no pulmonary embolism. An echocardiography showed a slight elevation of the systolic pulmonary artery pressure leading to the diagnosis of a possible diastolic dysfunction consecutive to a hypertensive cardiopathy. The patient's medical treatment for systemic hypertension was reinforced and no further explorations were conducted.

Due to disabling dyspnoea, the patient was hospitalised 4 months later. No signs of cardiac dysfunction were seen, the pulmonary auscultation was clear. Pulmonary embolism was eliminated by a pulmonary angiographic CT but rare ground-glass opacities were noticed. The echocardiography revealed signs of chronic cor pulmonale with dilatation of the right cavities and severe elevation of the systolic pulmonary artery pressure at $70 \mathrm{mmHg}$. The bronchoalveolar lavage 


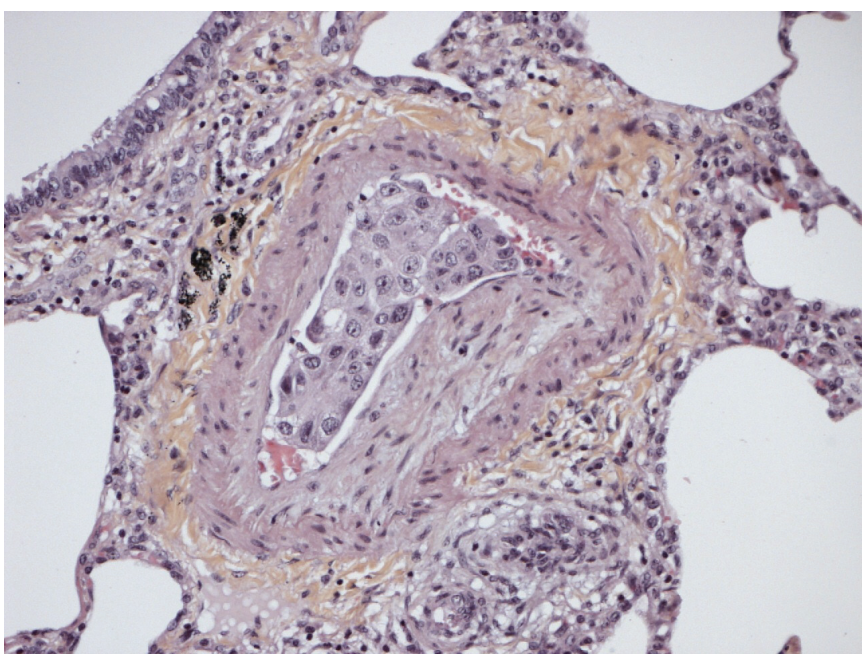

FIGURE 1. Fibromuscular intimal thickening leading to reduction of the lumen of the pulmonary arteriole. There is an accumulation of carcinomatous cells in the residual lumen. Haematoxylin and eosin stained, original magnification $\times 40$. showed intra-alveolar haemorrhage and no malignant cells, the pulmonary function tests were normal, the viral serologies and complete immune check-up were negative and the abdominal echography showed no sign of portal hypertension. Given the association of rapidly evolving pulmonary hypertension, alveolar haemorrhage and ground-glass opacities a possible pulmonary veno-occlusive disease was suspected [9]. The patient was addressed to our unit (Pneumology, Hospital CHU de Nantes, Nantes, France) for further investigations.

The right cardiac catheterisation revealed severe pulmonary hypertension with an elevation of mean artery pulmonary pressure at $62 \mathrm{mmHg}$, diminution of the cardiac output $\left(3.36 \mathrm{~L} \cdot \mathrm{min}^{-1}\right)$ and of the cardiac index $\left(2 \mathrm{~L} \cdot \mathrm{min}^{-1} \cdot \mathrm{m}^{-2}\right)$, and an increase of pulmonary vascular resistance at 16.7 Wood units. The pulmonary wedge pressure was not elevated $(6 \mathrm{mmHg})$. No cytologic study was performed during the procedure. The patient was transferred to the intensive care unit because of the appearance of low blood pressure and loss of consciousness. Treatment with dobutamine, noradrenalin, epoprostenol and nitric oxide was initiated, without improvement. A thrombocytopenia interpreted as a sign of severe pulmonary hypertension
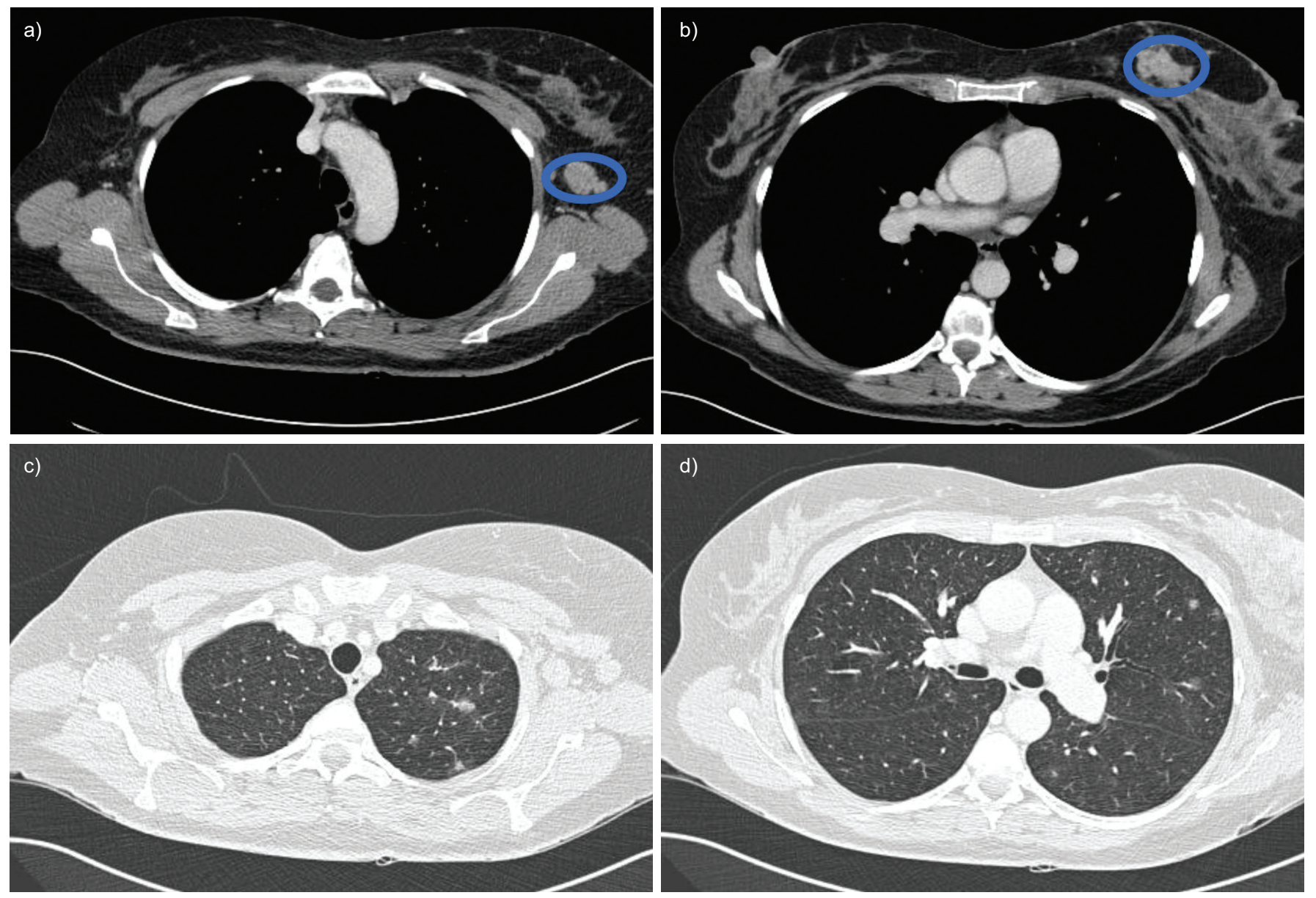

FIGURE 2. Pre-transplantation contrast enhanced computed tomography images showing a lesion of the left breast, a left axillary adenopathy and rare ground-glass nodules. a) Mediastinal window cut showing the left axillary adenopathy. b) Mediastinal window cut showing the lesion of the left breast. c and d) Parenchyma window cuts showing rare ground-glass nodules. 
appeared on the blood test. Given the dramatic evolution with signs of severe low cardiac flow the patient was put under venoarterial extracorporeal membrane oxygenation after oro-tracheal intubation and inscribed on an emergency transplantation list. She benefited from a cardiopulmonary transplantation 6 days after admission.

Rapidly a graft dysfunction associated with a multiorgan failure was observed. The anatomo-pathological examination of the explanted lungs showed tumoural cells within the pulmonary arteries associated with extensive intimal fibrosis consistent with PTTM (fig. 1). Following physical examination, an abnormality of the left breast and an axillary left lymphadenopathy were found. Blood tests showed a high level of the tumour marker CA 19-9. The CT scan confirmed a growth in the left breast associated with bilateral axillaries adenopathies and a left supraclavicular adenopathy. Biopsies of these lesions were performed revealing breast adenocarcinoma. A medullar invasion explaining the initial thrombocytopenia was revealed by a bone marrow examination.

The poor prognosis led to limiting specific care. The patient deceased 17 days after transplantation.

This case is important because breast cancer was not diagnosed due to the rapid evolution of the pulmonary hypertension and the need for emergency transplantation. Pulmonary hypertension was the first and only sign of malignancy. Neoplasia was not evoked due to the young age of the patient, absence of selfreported clinical signs and no specific findings on the CT scan. Retrospectively, it was found that a mammography conducted a few months earlier showed a lesion on the left breast (ACR 2). A new evaluation of the pre-transplant CT scan revealed a lesion of the left breast and a left axillary adenopathy (fig. 2).

The case also highlights the importance, for all patients, of a complete check-up before transplantation, including the search for a neoplasia. Indeed, when dealing with severe pulmonary hypertension, the possibility of a malignancy should always be kept in mind as it can lead to transplantation being reconsidered. In this case, the check-up failed to show any signs of malignancy even though the CT scan was pathological, emphasising the importance of a rigorous and complete analysis of the various imaging. The patient should have benefited from a gynaecological consultation as normally conducted before any transplantation but the rapid respiratory failure resulted in an incomplete pre-transplant evaluation. Moreover, development of a subacute pulmonary hypertension with rapid evolution towards right cardiac failure may be an indicator of malignancy.

Consequently, neoplasia should be considered when faced with any unexplained, rapidly developing, pulmonary hypertension.
Tania Madjer*, Isabelle Danner-Boucher*, Delphine Horeau-Langlard*, Alain Haloun*, Thierry Lepoivre", Christine Sagan and Antoine Magnan*

*INSERM UMR 915, Université de Nantes, CHU de Nantes, l'institut du thorax, Service de pneumologie, Plate-forme transversale d'allergologie, Centre de Référence National Maladies Rares Mucoviscidose, "CHU de Nantes, service d'anesthésie Réanimation, and ' $\mathrm{CHU}$ de Nantes, service d'anatomie pathologique, Nantes, France.

Correspondence: T. Madjer, Service de pneumologie de l'Hôpital Nord Laennec, Boulevard Jacques Monod, 44093, Nantes Cedex 1, France. E-mail: tania.madjer2@free.fr

Statement of Interest: A statement of interest for D. HoreauLanglard can be found at www.erj.ersjournals.com/site/misc/ statements.xhtml

\section{REFERENCES}

1 Roberts KE, Hamele-Bena D, Saqi A, et al. Pulmonary tumor embolism, a review of the literature. Am J Med 2003; 115: 228-232.

2 Chinen K, Tokuda Y, Fujiwara M, et al. Pulmonary tumor thrombotic microangiopathy in patients with gastric carcinoma: an analysis of 6 autopsy cases and review of the literature. Pathol Res Pract 2010; 206: 122-127.

3 Kuhnert C, Zeca E, Fischer J, et al. Hypertension arterielle pulmonaire par embolisation de cellules tumorales [Pulmonary hypertension due to tumor embolism]. Rev Med Interne 2010; 31: e6-8.

4 Liang YH, Kuo SW, Lin YL, et al. Disseminated microvascular pulmonary tumor embolism from non-small cell lung cancer leading to pulmonary hypertension followed by sudden cardiac arrest. Lung Cancer 2011; 72: 132-135.

5 Konduri S, Khan Q, Stites S. Pulmonary hypertension caused by metastatic breast cancer and its response to antihormone therapy and chemotherapy. Breast J 2007; 13: 506-508.

6 Wilson MK, Granger EK, Preda VA. Pulmonary hypertension due to isolated metastatic squamous cell carcinoma thromboemboli. Heart Lung Circ 2006; 15: 143-146.

7 Sancho-Chust JN, Ferrere J, Pineda J, et al. Pulmonary tumor embolism as an initial manifestation of pancreatic adenocarcinoma. Respir Care 2009; 54: 1732-1735.

8 Chinen K, Fujino T, Horita A, et al. Pulmonary tumor thrombotic microangiopathy caused by an ovarian cancer expressing tissue factor and vascular endothelial growth factor. Pathol Res Pract 2009; 205: 63-66.

9 Montani D, Price LC, Dorfmuller P, et al. Pulmonary veno-occlusive disease. Eur Respir J 2009; 33: 189-200.

DOI: $10.1183 / 09031936.00182711$ 